PROCEEDINGS OF THE

AMERICAN MATHEMATICAL SOCIETY

Volume 125, Number 9, September 1997, Pages 2759-2765

S 0002-9939(97)03895-

\title{
TOPOLOGICAL ENTROPY \\ FOR GEODESIC FLOWS ON FIBRE BUNDLES OVER RATIONALLY HYPERBOLIC MANIFOLDS
}

\author{
GABRIEL P. PATERNAIN
}

(Communicated by Mary Rees)

\begin{abstract}
Let $M$ be the total space of a fibre bundle with base a simply connected manifold whose loop space homology grows exponentially for a given coefficient field. Then we show that for any $C^{\infty}$ Riemannian metric $g$ on $M$, the topological entropy of the geodesic flow of $g$ is positive. It follows then, that there exist closed manifolds $M$ with arbitrary fundamental group, for which the geodesic flow of any $C^{\infty}$ Riemannian metric on $M$ has positive topological entropy.
\end{abstract}

\section{INTRODUCTION}

Let $M$ be a closed connected $C^{\infty}$ manifold and for a $C^{r}$ Riemannian metric $g$ $(r \geq 2)$, let $\phi_{t}: S M \rightarrow S M$ denote the geodesic flow of $g$, acting on the unit sphere bundle $S M$. Let $h_{\text {top }}(g)$ denote the topological entropy of the flow $\phi_{t}$.

For a compact simply connected manifold $X$, let $\Omega X$ denote the based loop space of $X$. We will say that $X$ is rationally hyperbolic if the sequence $\sum_{i=0}^{n} \operatorname{dim} H_{i}(\Omega X, \mathbf{Q})$ grows exponentially with $n$. The manifold $X$ is called rationally elliptic if the sequence $\sum_{i=0}^{n} \operatorname{dim} H_{i}(\Omega X, \mathbf{Q})$ grows subexponentially with $n$. It is well known that subexponential growth of the sequence $\sum_{i=0}^{n} \operatorname{dim} H_{i}(\Omega X, \mathbf{Q})$ implies polynomial growth (see $[2,3,7]$ and references therein).

"Generically" a simply connected compact manifold is rationally hyperbolic. Ellipticity imposes very strong restrictions. In particular, if $X^{n}$ is rationally elliptic then $\operatorname{dim} H_{*}(X, \mathbf{Q}) \leq 2^{n}[7,8]$. It follows that if $W$ is any simply connected compact manifold that is not a rational homology sphere, then the connected sum of a large enough number of copies of $W$ must be rationally hyperbolic. In the case of $W=S^{k} \times S^{l}$, with $k, l \geq 2,\left(S^{k} \times S^{l}\right) \#\left(S^{k} \times S^{l}\right)$ is already rationally hyperbolic [9].

Our aim will be to study the topological entropy of the geodesic flow of a Riemannian metric on a fibre bundle whose base space is rationally hyperbolic, or more generally, a space whose loop space homology grows exponentially for a given coefficient field. More precisely we shall show:

Received by the editors April 6, 1995 and, in revised form, August 3, 1995, and March 22, 1996.

1991 Mathematics Subject Classification. Primary 58F17, 58E10.

Key words and phrases. Geodesic flow, topological entropy, loop space homology.

Supported by grants from CSIC and CONICYT.

(C) 1997 American Mathematical Society 
Theorem 1.1. Suppose $M$ is the total space of a fibre bundle with base a compact simply connected manifold whose loop space homology grows exponentially for a given coefficient field. Then for any $C^{\infty}$ Riemannian metric on $M, h_{\text {top }}(g)>0$.

We shall describe now two consequences of Theorem 1.1. Recall that if $G$ is a finitely presented group, then there exists a closed 4-manifold $N$ so that $\pi_{1}(N)=G$ [11]. Therefore by considering the 8-manifold $M=\left(\left(S^{2} \times S^{2}\right) \#\left(S^{2} \times S^{2}\right)\right) \times N$, Theorem 1.1 implies:

Corollary 1.2. Let $G$ be any finitely presented group. Then there exists a closed 8-manifold $M$ with $\pi_{1}(M)=G$, so that for any $C^{\infty}$ Riemannian metric $g$ on $M$, $h_{\text {top }}(g)>0$.

Given a pair of points $(p, q) \in M \times M$, define $n(p, q, \lambda)$ as the number of geodesic segments of length $\leq \lambda$ connecting $p$ and $q$. It is not hard to see that, for each $\lambda>0$, the counting function $n(p, q, \lambda)$ is finite and locally constant on an open full measure subset of $M \times M$, and integrable on $M \times M$. In [10], R. Mañé showed that:

$$
\lim _{\lambda \rightarrow \infty} \frac{1}{\lambda} \log \int_{M \times M} n(p, q, \lambda) d p d q=h_{t o p}(g) .
$$

Thus Theorem 1.1 combined with Mañé's result implies the following:

Corollary 1.3. Suppose $M$ is the total space of a fibre bundle with base a compact simply connected manifold whose loop space homology grows exponentially for a given coefficient field. Then for any $C^{\infty}$ Riemannian metric on $M$, there are on average exponentially many geodesics between two points.

The main novelty in Theorem 1.1 and Corollary 1.2 is that there are no restrictions on the fundamental group of $M$. A classical result of Dinaburg [1] asserts that if $\pi_{1}(M)$ has exponential growth, then $h_{t o p}(g)>0$, for any $C^{2}$ metric $g$ on $M$. If $M$ itself is simply connected and for a given coefficient field its loop space homology grows exponentially, then $h_{t o p}(g)>0$ for any $C^{\infty}$ Riemannian metric $g$; this fact was pointed out by Gromov in [4, Section 2.7] (a detailed proof is given in $[13$, Theorem 3.2]). The result of the present paper is closely related to this result of Gromov.

The key idea behind Gromov's proof is the combination of Yomdin's Theorem [15] with a relationship between the size of the homology of the loop space and the number of geodesics joining two points on the manifold obtained in [5]. However for manifolds with infinite fundamental group the growth of the homology of any of the components of the loop space does not seem to relate clearly with the growth of the average number of geodesics between two points on the manifold: Gromov's arguments in [5] are no longer valid (this is explicitly mentioned in [6, p. 104]). We avoid this difficulty in the case of a fibre bundle, by considering the space of paths leaving from a point and ending in a fixed fibre, and applying Yomdin's Theorem to the normal sphere bundle of the latter.

Note that if $M$ is simply connected and it fibres over a manifold $X$ as in Theorem 1.1, then the loop space homology of $M$ also grows exponentially for some coefficient field. This follows from the spectral sequence of the fibration $\Omega M \rightarrow \Omega(p, N) \rightarrow N$, where $N$ denotes the fibre of $M \rightarrow X$, and $\Omega(p, N)$ is the space of paths leaving from a point $p \in M$ and ending in a fixed fibre (if the homology of the loop space of $X$ grows exponentially, the homology of $\Omega(p, N)$ also grows exponentially). 


\section{Proof of Theorem 1.1}

Let $N$ denote the fibre of the fibration $\pi: M \rightarrow X$. For a point $y \in X$, let $N_{y}=\pi^{-1}(y) \cong N$. For a point $y \in X$ and a point $p \in M$, let $\Omega\left(p, N_{y}\right)$ denote the space of piecewise smooth paths defined on $[0,1]$ leaving from $p$ and ending in $N_{y}$. Also, for $x, y \in X$, let $\Omega(X, x, y)$ denote the space of piecewise smooth paths in $X$ defined on $[0,1]$ from $x$ to $y$. Recall that the homotopy type of $\Omega(X, x, y)$ is independent of the points $x$ and $y$.

Now let $g$ be an arbitrary $C^{\infty}$ Riemannian metric on $M$ and let $L_{g}(\alpha)$ denote the length of a path in $M$. As usual, $\Omega^{\lambda}\left(p, N_{y}\right)$ will denote the subspace of paths $\alpha$ such that $L_{g}(\alpha) \leq \lambda$.

Our theorem will be a consequence of the following proposition (compare with [5] and [6, p. 102]).

Proposition 2.1. There exists a constant $C>0$ such that given any point $p \in M$ and given any positive integer $i$, we have

$$
\operatorname{dim} H_{i}(\Omega X) \leq \operatorname{dim} H_{i}\left(\Omega^{l}\left(p, N_{y}\right)\right),
$$

for any $l \geq C i$.

Proof. We shall use the following result due to Gromov [5]. A proof is given in the appendix.

Theorem 2.2. Given a metric $g_{X}$ on $X$ there exists a constant $C_{1}>0$ such that given any pair of points $x$ and $y$ in $X$ and any positive integer $i$, any element in $H_{i}(\Omega(X, x, y))$ can be represented by a cycle whose image lies in $\Omega^{C_{1} i}(X, x, y)$.

Observe that $\pi: M \rightarrow X$ naturally induces a map $\hat{\pi}: \Omega\left(p, N_{y}\right) \rightarrow \Omega(X, \pi(p), y)$.

Proposition 2.3. Let $g$ and $g_{X}$ be metrics on $M$ and $X$ respectively. For any $p \in M$ and $y \in X$, there is a continuous map

$$
\theta: \Omega(X, \pi(p), y) \rightarrow \Omega\left(p, N_{y}\right)
$$

such that $\hat{\pi} \circ \theta$ is the identity and there is a constant $B>0$ such that

$$
L_{g}(\theta(\omega)) \leq B L_{g_{X}}(\omega)
$$

for any $\omega \in \Omega(X, \pi(p), y)$. The constant $B$ depends only on $g$ and $g_{X}$ and is independent of $p, y$ and $\omega$.

Proof. Choose a metric $g_{M}$ on $M$ such that

$$
\pi:\left(M, g_{M}\right) \rightarrow\left(X, g_{X}\right)
$$

is a Riemannian submersion. The Riemannian metric $g_{M}$ naturally defines a horizontal distribution and the horizontal lift of a piecewise smooth path on $X$ is uniquely defined provided an initial point in $M$ is chosen. Therefore there exists a continuous map $\theta: \Omega(X, \pi(p), y) \rightarrow \Omega\left(p, N_{y}\right)$ with $\hat{\pi} \circ \theta=i d$.

Since $M$ is compact, there exists a constant $B>0$ such that if $\alpha$ is any piecewise smooth path in $M$, then

$$
L_{g}(\alpha) \leq B L_{g_{M}}(\alpha) .
$$

Now let $\omega$ be a path in $\Omega(X, \pi(p), y)$. Then

$$
L_{g}(\theta(\omega)) \leq B L_{g_{M}}(\theta(\omega))=B L_{g_{X}}(\omega)
$$

since $\pi:\left(M, g_{M}\right) \rightarrow\left(X, g_{X}\right)$ is a Riemannian submersion and $\theta$ is the associated horizontal lift. 
Let us complete the proof of Proposition 2.1. Take $p$ in $M$, and let $x=\pi(p)$. Let $C=C_{1} B$, where $C_{1}$ is the constant given by Theorem 2.2 and $B$ is given by Proposition 2.3. Set $A=\Omega^{l}\left(p, N_{y}\right)$ for $l \geq C i$. The map

$$
\hat{\pi}_{A}=\hat{\pi}_{\mid A}: A \rightarrow \Omega(X, x, y)
$$

naturally induces a morphism

$$
\left(\hat{\pi}_{A}\right)_{*}: H_{i}(A) \rightarrow H_{i}(\Omega(X, x, y)) .
$$

To prove the proposition it suffices to show that this morphism is onto. Take $\eta \in H_{i}(\Omega(X, x, y))$. By Theorem 2.2, $\eta$ can be represented by a cycle $\zeta$ whose image lies in $\Omega^{C_{1} i}(X, x, y)$. By Proposition 2.3, $\theta \circ \zeta$ is a cycle whose image lies in $\Omega^{C_{1} B i}\left(p, N_{y}\right)$ and therefore it defines an element in $H_{i}(A)$. But $\left(\hat{\pi}_{A}\right)_{*}\left(\theta_{*}(\eta)\right)=\eta$ since $\hat{\pi} \circ \theta=i d$ which concludes the proof of the proposition.

Let us explain now how Theorem 1.1 can be derived from Proposition 2.1. If the point $p$ is not a focal point with respect to $N_{y}$, then $L_{g}: \Omega\left(p, N_{y}\right) \rightarrow \mathbf{R}$ is a Morse function, with critical points the geodesic segments leaving from $p$ and ending orthogonally in $N_{y}$. Let $n(p, \lambda)$ denote the number of these geodesic segments with length $\leq \lambda$. Then the Morse inequalities and Proposition 2.1 imply:

$$
\sum_{i=0}^{m} \operatorname{dim} H_{i}(\Omega X) \leq n(p, C m) .
$$

Integrating the last inequality with respect to $p$ we obtain:

$$
\sum_{i=0}^{m} \operatorname{dim} H_{i}(\Omega X) \leq \frac{1}{\operatorname{Vol}_{g}(M)} \int_{M} n(p, C m) d p .
$$

As in [14] we have that

$$
\limsup _{\lambda \rightarrow \infty} \frac{1}{\lambda} \log \int_{M} n(p, \lambda) d p \leq \limsup _{\lambda \rightarrow \infty} \frac{1}{\lambda} \log \operatorname{Vol}\left(\phi_{\lambda}\left(S N_{y}^{\perp}\right)\right),
$$

where $S N_{y}^{\perp}$ denotes the normal sphere bundle of $N_{y}$. On the other hand, Yomdin's Theorem [15] implies,

$$
\limsup _{\lambda \rightarrow \infty} \frac{1}{\lambda} \log \operatorname{Vol}\left(\phi_{\lambda}\left(S N_{y}^{\perp}\right)\right) \leq h_{t o p}(g) .
$$

From the last three inequalities and the fact that $\sum_{i=0}^{m} \operatorname{dim} H_{i}(\Omega X)$ grows exponentially with $m$, it follows right away that $h_{t o p}(g)>0$, which concludes the proof of Theorem 1.1.

Remark 2.4. Note that we have proved in fact a stronger result: the topological entropy of the geodesic flow of $g$ with respect to the normal sphere bundle of any fibre is positive.

\section{Appendix}

In this appendix we shall prove Theorem 2.2. Gromov's original proof in [5] is very short. A more detailed proof is given in [6, p. 102] but it contains some mistakes.

Let $\left\{V_{\alpha}\right\}$ be a finite covering of $X$ by convex open sets. Recall that a set is convex if any two points in it are joined by a unique geodesic that stays in the set. Choose a triangulation $T$ of $X$ that is fine enough so that each closed simplex of $T$ lies in one of the $V_{\alpha}$. We shall also assume that the 1-skeleton of $T$ consists of 
geodesic segments. For each point $p \in X$, let $T(p)$ be the (closed) face of $T$ of minimum dimension that contains $p$ (so $T(p)=\{p\}$ if $p$ is a vertex of $T$ ) and let $O(p)$ be the union of all maximal simplices of $T$ that contain $p$.

Given a positive integer $k$, we define open subsets $\Omega_{k}(X, x, y)$ of $\Omega(X, x, y)$ in the following way. We shall say that $\omega \in \Omega_{k}(X, x, y)$ if for each integer $j=1,2, \ldots, 2^{k}$, the image under $\omega$ of each subinterval $\left[(j-1) / 2^{k}, j / 2^{k}\right]$ lies in one of the sets $V_{\alpha}$ and

lies in the same $V_{\alpha}$.

$$
\left.O\left(\omega\left((j-1) / 2^{k}\right)\right)\right) \cup O\left(\omega\left(j / 2^{k}\right)\right)
$$

Let $B_{k}(X, x, y) \subset \Omega_{k}(X, x, y)$ be the space of broken geodesics $\gamma$ such that $\gamma \in \Omega_{k}(X, x, y)$ and the restriction of $\gamma$ to each subinterval $\left[(j-1) / 2^{k}, j / 2^{k}\right]$ is a geodesic parameterized at constant speed. Each $\gamma \in B_{k}(X, x, y)$ determines a sequence $\left\{p_{j}=\gamma\left(j / 2^{k}\right)\right\}$ which has the properties:

(1) $p_{0}=x$ and $p_{2^{k}}=y$;

(2) $O\left(p_{j-1}\right) \cup O\left(p_{j}\right)$ lies in a single $V_{\alpha}$ for each $j=1,2, \ldots, 2^{k}$.

Conversely any sequence $\left\{p_{j}\right\}, 0 \leq j \leq 2^{k}$, with these two properties determines a broken geodesic in $B_{k}(X, x, y)$. Moreover the correspondence between broken geodesics in $B_{k}(X, x, y)$ and sequences of points is bijective (because the parameterization will distinguish different geodesics with the same trace). Observe that this correspondence induces on $B_{k}(X, x, y)$ a cell decomposition: a cell that contains $\gamma$ is given by the cartesian product

$$
T\left(p_{1}\right) \times T\left(p_{2}\right) \times \cdots \times T\left(p_{2^{k}-1}\right) .
$$

Thus we can think of $B_{k}(X, x, y)$ as a finite cell complex. Note that the methods in Milnor's book [12] show that $B_{k}(X, x, y)$ is a deformation retract of $\Omega_{k}(X, x, y)$.

Since $X$ is simply connected, there exists a smooth map $f: X \rightarrow X$ such that $f$ collapses the 1 -skeleton of the triangulation $T$ to a point and $f$ is smoothly homotopic to the identity. Observe that $f$ naturally induces a map $\hat{f}: \Omega(X, x, y) \rightarrow$ $\Omega(X, f(x), f(y))$.

We need the following lemma.

Lemma 3.1. There exists a constant $C_{1}>0$ such that for any integer $k \geq 1$, we have

$$
\hat{f}\left(i \text {-skeleton of } B_{k}(X, x, y)\right) \subset \Omega^{C_{1} i}(X, f(x), f(y)),
$$

for all $i \leq \operatorname{dim} B_{k}(X, x, y)$.

Proof. Consider a cell

$$
T\left(p_{1}\right) \times T\left(p_{2}\right) \times \cdots \times T\left(p_{2^{k}-1}\right)
$$

with dimension $i \leq \operatorname{dim} B_{k}(X, x, y)$. Take a path $\gamma$ in this cell. Then $\gamma$ is a broken geodesic, each leg of which lies in one of the sets $V_{\alpha}$. Since $f$ sends the 1-skeleton of the triangulation $T$ to a point we observe that

$$
L_{g_{X}}(\hat{f}(\gamma)) \leq K \cdot d \cdot N(\gamma),
$$

where $K \stackrel{\text { def }}{=} \max _{x \in X}\left\|d_{x} f\right\|, d$ is the maximum of the $g_{X}$-diameters of all the convex open sets $V_{\alpha}$, and $N(\gamma)$ is the number of legs of $\gamma$ that do not lie in the 1-skeleton. Since the 1-skeleton of the triangulation $T$ is made up of geodesic segments, the leg of the broken geodesic $\gamma$ that joins $T\left(p_{j}\right)$ to $T\left(p_{j+1}\right)$ must lie in the 1-skeleton if $1 \leq j<2^{k}-1$ and $\operatorname{dim} T\left(p_{j}\right)=\operatorname{dim} T\left(p_{j+1}\right)=0$. Thus the only legs of $\gamma$ that could fail to lie in the 1 -skeleton are the initial leg, which starts at $x$, the final leg, 
which ends at $y$, and legs that begin or end in a $T\left(p_{j}\right)$ with nonzero dimension. We see that

$$
N(\gamma) \leq 2+2 i \leq 4 i
$$

If we set $C_{1}=4 K d$ we obtain

$$
L_{g_{X}}(\hat{f}(\gamma)) \leq C_{1} i
$$

which concludes the proof of the lemma.

We shall show that for all $x$ and $y$ in $X$, any $\eta \in H_{i}(\Omega(X, f(x), f(y))$ can be represented by a cycle whose image lies in $\Omega^{C_{1} i}(X, f(x), f(y))$, where $C_{1}$ is the constant given by Lemma 3.1. This implies the theorem since $f$ is a surjective map (it has degree one since it is homotopic to the identity).

Observe that

$$
\Omega_{1}(X, x, y) \subset \Omega_{2}(X, x, y) \subset \Omega_{3}(X, x, y) \subset \ldots
$$

and

$$
\Omega(X, x, y)=\bigcup_{k=1}^{\infty} \Omega_{k}(X, x, y) .
$$

Since $f$ is homotopic to the identity, there exists $\mu \in H_{i}(\Omega(X, x, y))$ such that $\hat{f}_{*}(\mu)=\eta$. If we are given a cycle that represents $\mu$, this cycle will have an image that lies in $\Omega_{k}(X, x, y)$ for some $k$. Retract $\Omega_{k}(X, x, y)$ onto $B_{k}(X, x, y)$. Then our cycle can be moved by a homotopy into the $i$-skeleton of $B_{k}(X, x, y)$. By Lemma 3.1, $\hat{f}$ maps all points in the $i$-skeleton of $B_{k}(X, x, y)$ to points in $\Omega^{C_{1} i}(X, f(x), f(y))$ and hence $\hat{f}_{*}(\mu)=\eta$ can be represented by a cycle whose image lies in $\Omega^{C_{1} i}(X, f(x), f(y))$.

\section{ACKNOWLEDGMENT}

I would like to thank Karsten Grove for several useful discussions and the referee for an extremely detailed report, which considerably improved this paper.

\section{REFERENCES}

[1] E. I. Dinaburg, On the relations among various entropy characteristics of dynamical systems, Math USSR Izv. 5 (1971) 337-378.

[2] Y. Félix, S. Halperin, J.C. Thomas, Elliptic spaces, Bull. AMS 25 (1991) 69-73. MR 92m:55009

[3] Y. Félix, S. Halperin, J.C. Thomas, Elliptic spaces II, L'Enseignement Mathèmatique 39 (1993) 25-32. MR 94f:55008

[4] M. Gromov, Entropy, homology and semialgebraic Geometry, Séminarie Bourbaki 38éme année, 1985-86, No 663, 225-240. MR 89f:58082

[5] M. Gromov, Homotopical effects of dilatation, J. Diff. Geom. 13 (1978) 303-310. MR 82d:58017

[6] M. Gromov, Structures métriques pour les variétés Riemanniennes, by J. Lafontaine and P. Pansu, Editions Cedic/Fernand Nathan, Paris, 1981. MR 85e:53051

[7] K. Grove, S. Halperin, Contributions of Rational Homotopy Theory to global problems in Geometry, Publ. Math. I.H.E.S. 56 (1982), 379-385. MR 84b:58030

[8] S. Halperin, Spaces whose rational homology and De Rham homotopy are both finite dimensional, Astérisque, 113-114 (1984) 198-205. MR 86a:55015

[9] S. Halperin, J. M. Lemaire, Suites inertes dans les algèbres de Lie gradues, Math. Scand. 61 (1987) 39-67. MR 89e:55022

[10] R. Mañé, On the topological entropy of geodesic flows, preprint, IMPA, 1994.

[11] W. S. Massey, Algebraic Topology: An Introduction, Springer-Verlag, New York, 1978. 
[12] J. Milnor, Morse Theory, Ann. of Math. Studies 51, Princeton Univ. Press: Princeton, NJ, 1969.

[13] G. P. Paternain, On the topology of manifolds with completely integrable geodesic flows, Ergod. Th. and Dyn. Syst. 12 (1992), 109-121. MR 93g:58117

[14] G. P. Paternain, M. Paternain, Topological entropy versus geodesic entropy, International Journal of Math. 2 (1994) 213-218. MR 95h:58101

[15] Y. Yomdin, Volume growth and entropy, Israel J. Math. 57 (1987), 287-300. MR 90g:58008

imerl-Facultad de Ingeniería, Julio Herrera y Reissig 565, C.C. 30, Montevideo, URUGUAY

E-mail address: gabriel@cmat.edu.uy 